\title{
DESENVOLVIMENTO E IMPLANTAÇÃO DE UM SISTEMA INTELIGENTE DE MONITORAMENTO DE CABOS DE ESTAIAMENTO PARA EVITAR SINISTROS COM QUEDA DE TORRES DE TRANSMISSÃO DE ENERGIA
}

\begin{abstract}
Alex de Lima e Silva
Doutorando em Engenharia Elétrica - Eletrônica de Potência, pesquisador, Rio de Janeiro, Brasil prof.alexdelima@gmail.com

Fabrício Neves

Engenheiro Eletricista, pesquisador, Rio de Janeiro, Brasil engenharia@geteceng.com.br

José Alves da Silva Júnior Engenheiro Eletricista, pesquisador, Rio de Janeiro, Brasil jose.alves@geteceng.com.br

Tallis Amorim Simões Mestre em Engenharia Elétrica, pesquisador, Rio de Janeiro, Brasil tallis_as@hotmail.com

André Felipe A. Marandino Guimarães Engenheiro Eletricista, pesquisador, Rio de Janeiro, Brasil afamguimaraes@poli.ufrj.br
\end{abstract}

\begin{abstract}
RESUMO
O presente artigo aborda os aspectos técnicos associados ao desenvolvimento de um sistema inteligente de detecção e monitoramento para evitar sinistros com queda de torres de transmissão de energia. O sistema desenvolvido é composto, dentre outros, por um conjunto de sensores conectados aos cabos de torres estaiadas e por um sistema de telecomunicação, que envia os dados para um Controlador Lógico Programável. Este controlador é responsável por inferir se a operação do cabo da torre de transmissão é normal ou anormal e apresentar esta informação ao usuário por meio de uma Interface Homem Máquina integrada ao painel. Outros aspectos abordados são os sistemas periféricos associados, tais como o sistema de suprimento de energia, composto por painéis fotovoltaicos e um banco de baterias que garantem o funcionamento do sistema de detecção de avarias por até uma semana. O uso deste sistema permitirá à concessionária de transmissão identificar em tempo real a ocorrência de situações de riscos nas torres de suas linhas de transmissão, permitindo direcionar o esforço da equipe de manutenção e evitar danos mais graves aos seus ativos.
\end{abstract}

Palavras-chave: Sistema de Transmissão. Monitoramento de Cabo Estaiado. Energia Solar. Manutenção de Linhas de Transmissão.

\section{DEVELOPMENT AND IMPLEMENTATION OF AN INTELLIGENT SYSTEM FOR GUYED CABLE MONITORING TO AVOID CASUALTY WITH COLLAPSE OF TRANSMISSION LINE'S TOWERS ABSTRACT}


This article approaches the aspects related with the development of an intelligent system for detection and monitoring to avoid casualty with collapse of transmission line's towers. A group of sensors are located on the guyed cables of the transmission line's tower and a communication system acts exchanging information with Programmable Logic Controller. This controller infers if the operation o the cables is normal or not and shows this information on a Human Machine Interface. Some other aspects, such as the power supply done by batteries bank and a photovoltaic system, are addressed in this work. This system allows the transmission companies a faster identification of situations where its assets are operating under risk of damage, providing information in a way that the company may send out it's maintenance team to deal with the situation.

Keywords: Transmission System. Stay Cable Monitoring. Transmission Line Maintenance. Solar Energy.

\section{INTRODUÇÃO}

Um sistema elétrico de potência possui como objetivo suprir uma carga elétrica e aqueles de grande porte, podem ser subdivididos em geração, transmissão e distribuição. $O$ sistema brasileiro é um destes classificados como de grande porte (RAUPP, 2016), possuindo uma capacidade instalada de $161 \mathrm{GW}$ e um sistema de transmissão com mais de $141 \mathrm{mil}$ km de linhas de transmissão responsáveis por atender uma carga de 90 GW (ONS,2019). As concessionárias de transmissão são as empresas proprietárias dos equipamentos que compõe o sistema de transmissão, sendo responsáveis por operar e manter estes equipamentos que apresenta grandes desafios advindos dos longos comprimentos das linhas de transmissão.

As concessionárias de transmissão se utilizam de uma estrutura de manutenção compacta, geralmente dispersada no percurso de suas linhas de transmissão. Além disso, estas linhas de transmissão podem, por vezes, atravessar regiões de difícil acesso, como áreas de preservação permanente ou regiões urbanas densamente povoadas com risco associado à criminalidade, também chamadas de Áreas com Severas Restrições à Operação (HUBACK, 2018). Uma das consequências é que a inspeção periódica de todos os elementos que compõem uma linha de transmissão, especialmente as torres, acaba por ser realizada com intervalos longos inviabilizando a identificação de determinados problemas. Por este motivo, as concessionárias de transmissão têm investido cada vez mais recursos em sistemas capazes de monitorar remotamente o estado de seus ativos.

Uma das verificações realizadas pelas equipes de manutenção é o estado dos cabos que sustentam as torres estaiadas. A ocorrência de danos e avarias nestes cabos pode acarretar na queda da torre e, consequentemente, no desligamento deste elemento do sistema elétrico por um longo período. Por este motivo, as concessionárias de transmissão têm investido seus métodos e 
sistemas para monitoramento remoto do estado de seus ativos, tais como os descritos em (CLARO, 2009) e (ROSSI, 2009).

A Norte Brasil Transmissora de Energia S.A. - NBTE, é detentora de parte do sistema de transmissão que interliga as usinas do Rio Madeira, na região Norte do Brasil, ao centro de carga do país na região sudeste. Possui $2.411 \mathrm{~km}$ de linhas de transmissão composta por 4340 torres. Esta concessionária relatou que no período de ano foram registrados diversos atos de vandalismo, acarretando em quedas de 15 estruturas e avarias à outras 6, o que acarreou em grandes prejuízos financeiros à esta concessionária. Conforme (LIMA, 2012) uma das maiores causas de sinistros em torres no brasil é o vandalismo, tais como retirada ou corte de estais.

Tendo em vista a gravidade dos eventos de queda de torre de linha de transmissão, a concessionária de transmissão NBTE. e a empresa executora GETEC ENGENHARIA iniciaram o projeto de Pesquisa e Desenvolvimento (P\&D) apresentado neste artigo, cuja elaboração e execução atendem à Lei n 9.991/ 2000, ao manual de P\&D 2012, à Resolução Normativa (REN) ANEEL 504/2012 e à REN ANEEL 754/2016.

O projeto de P\&D em desenvolvimento trata-se de um sistema para monitorar e detectar danos em cabos estaiados de torres de linha transmissão com o propósito de identificar avarias, tais como tentativa de corte ou rompimento destes estais, de forma antecipada. O sistema apresenta, em tempo real, a informação de operação anômala dos elementos monitorados para uma Interface Homem Máquina, por meio da qual o usuário pode identificar qual equipamento está sob risco. Os equipamentos montados diretamente na torre são supridos por um sistema de geração fotovoltaico associado à um banco de baterias, capaz de manter o sistema embarcado na torre em operação por uma semana.

Este artigo contempla as principais etapas do projeto, desde sua concepção, ensaios laboratoriais, implantação e comissionamento em torre de transmissão de energia utilizada como modelo, concluindo o projeto de P\&D.

\section{DESENVOLVIMENTO}

O sistema proposto foi desenvolvido ao longo de seis etapas em que foi desenvolvido um protótipo de um sistema de detecção e monitoramento de cabos estaiados de torres de linhas de transmissão.

Os componentes deste sistema são os sensores que irão medir as vibrações nos cabos das torres, o sistema de telecomunicações que irá transmitir as informações dos sensores até o 
microprocessador Arduino, a lógica de monitoramento e controle implementara neste bem como a Interface Homem Máquina (IHM), além de um sistema de fornecimento de energia fotovoltaico para a parcela embarcada do sistema proposto.

Foram especificados e testados os sistemas de comunicação entre os componentes bem como as lógicas implementadas nos microcontroladores. Foram realizados ensaios laboratoriais e em campo dos protótipos montados com vistas a se obter informações a respeito do comportamento das medidas dos sensores frente às variadas formas de operação dos cabos monitorados. Além disso, foi verificada a capacidade e o desempenho do sistema de comunicação e o funcionamento do sistema desenvolvido como um todo, desde a aplicação da perturbação no cabo até a sinalização da operação anormal na IHM.

\subsection{SISTEMA DE TRANSMISSÃO E RECEPÇÃO DE DADOS}

O sistema de transmissão e recepção de dados é responsável por obter os dados dos sensores localizados nos cabos de estai da torre e enviá-los até o microcontrolador. Na Figura 1 estão os principais componentes deste sistema.

Figura 1 - Esquema básico do sistema de transmissão e recepção de dados

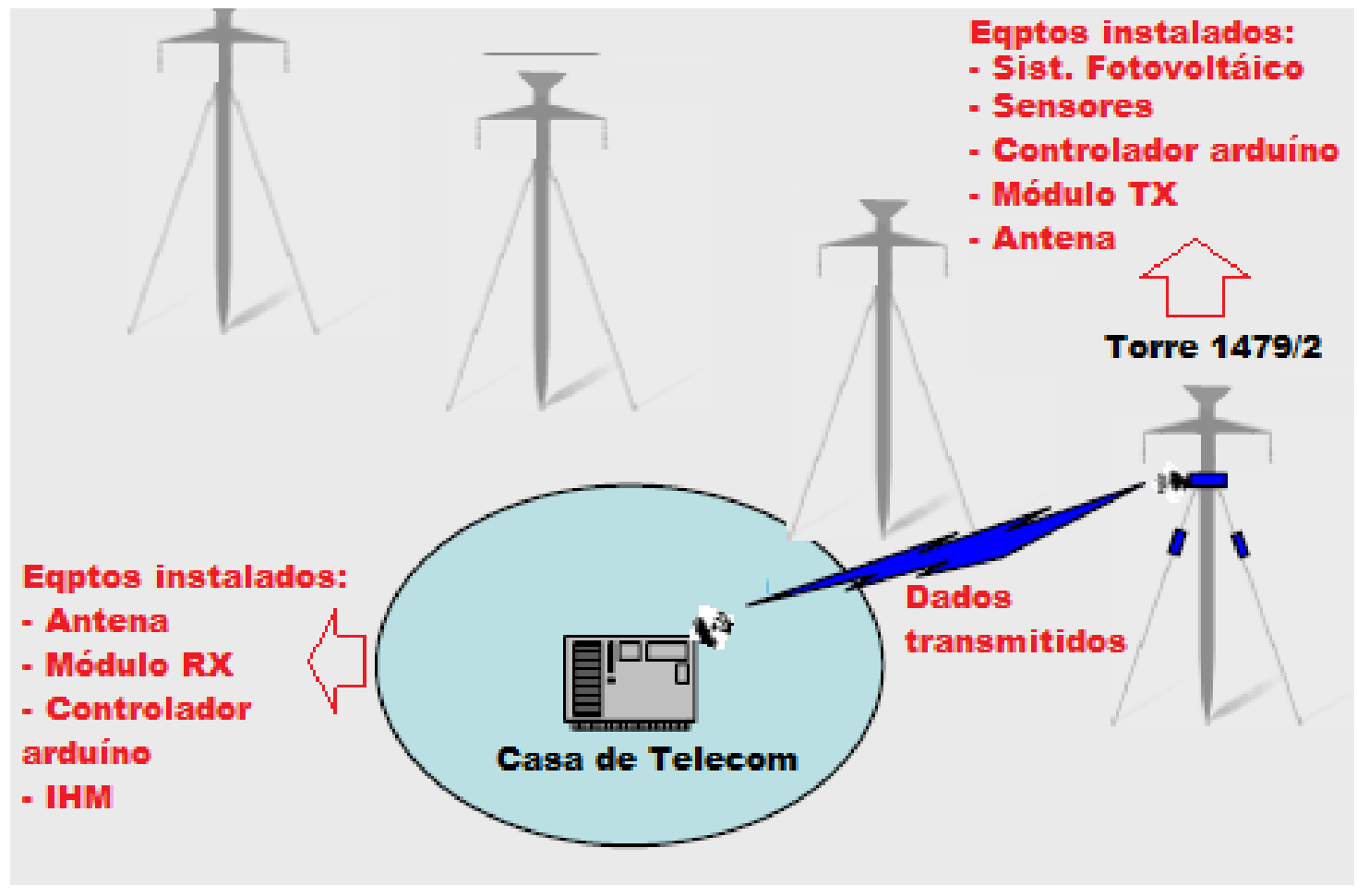

Fonte: (Adaptado de GETEC,2017) 
Os sensores empregados são do tipo MPU 6050, do tipo acelerômetro com giroscópio integrado, cuja saída de tensão varia entre 0 e 5V (INVESENSE, 2013). No sistema desenvolvido, são medidos valores de aceleração de até $8 \mathrm{G}$, sendo este valor o fundo de escala ideal para aplicação proposta.

Para comunicação entre o sistema embarcado na torre e os equipamentos instalados na Casal de Telecom, foi utilizado o transceiver APC220, que possui alcance de até $1 \mathrm{~km}$ em campo aberto e microcontrolador integrado que cria interface UART/TTL, permitindo a realização de transmissão de dados sem a necessidade de programação específica. Como este componente possui a capacidade de transmitir e receber dados, será possível futuramente enviar comandos e dados para o sistema embarcado na torre a partir da Casa de Telecom. Isso permitirá, por exemplo a execução de rotinas de diagnóstico dos sensores, acionamento de alarmes para afugentar vândalos ou até mesmo ajustar a posição das placas fotovoltaicas para melhor aproveitamento da luz solar.

Para alimentar os componentes do sistema embarcado na torre, utilizou-se uma solução composta por um sistema de geração fotovoltaica associada à um banco de baterias. Os equipamentos localizados na Casa de Telecom são atendidos por um banco de baterias que é carregado a partir de uma rede AC 127/220 V $\mathrm{V}_{\mathrm{AC}}$. Os dois conjuntos de banco de baterias são capazes de manter o sistema todo em funcionamento por até uma semana na hipótese de ausência ou falha no sistema de carregamento do banco de baterias.

A topologia do sistema de transmissão e recepção de dados é a ilustrada na Figura 2.

Figura 2 - Topologia do sistema de transmissão e recepção de dados.
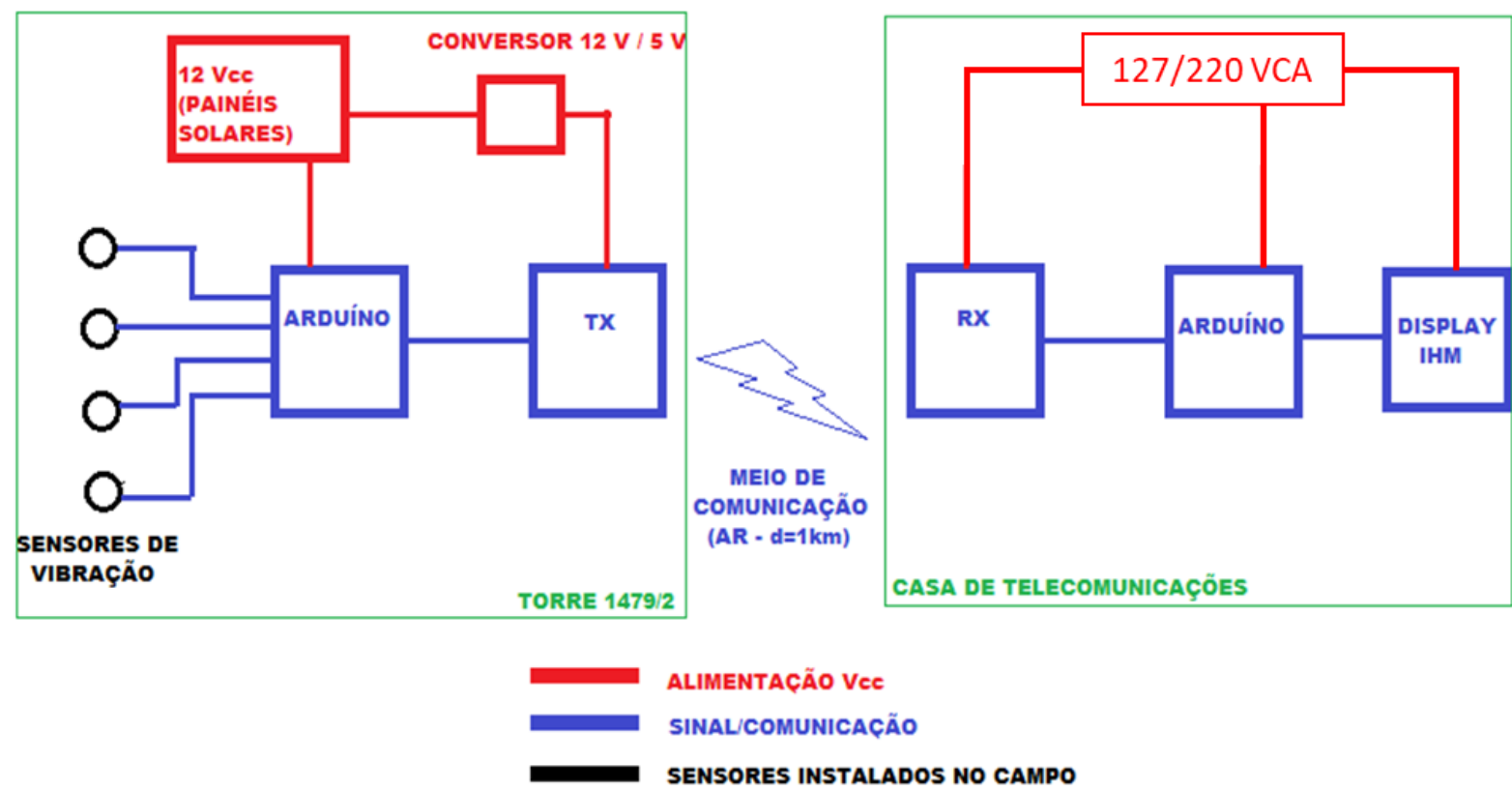

Fonte: (Autoria própria). 


\subsection{ESTRUTURA DO MICROCONTROLADOR}

O microcontrolador utilizado é do tipo Arduino modelo ATmega2560, no qual foi implementado um algoritmo que realiza uma rotina básica de leitura e comparação dos valores dos sensores com uma referência, uma segunda rotina de leitura e comparação do valor de tensão dos bancos de baterias e uma terceira função associada à recepção de informação e envio para a IHM.

A rotina principal é executada pelo microcontrolador embarcado na torre cujos estais estão sob monitoração. Esta rotina consiste em, ciclicamente, executar as sub-rotinas de análise de cada estai e banco de baterias, conforme fluxograma ilustrado na Figura 3.

Figura 3 - Fluxograma da função principal do algoritmo implementado no microcontrolador embarcado.

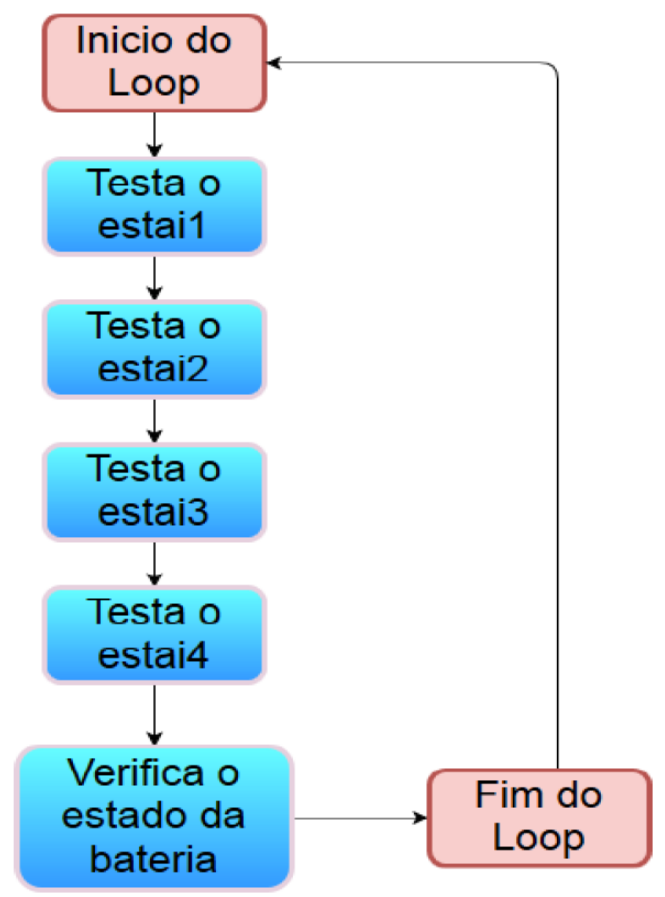

Fonte: (Autoria própria).

Cada uma das sub-rotinas do tipo "Testa o estai X" consistem em obter o valor do sensor correspondente ao estai, compará-lo com valores preestabelecidos e, dependendo da condição operativa constatada para o estai, emite-se um sinal de alarme para o microcontrolador da Casa de Telecom. Esta sub-rotina está ilustrada na Figura 4. 
Figura 4 - Sub-rotina de avaliação da condição operativa dos estais.

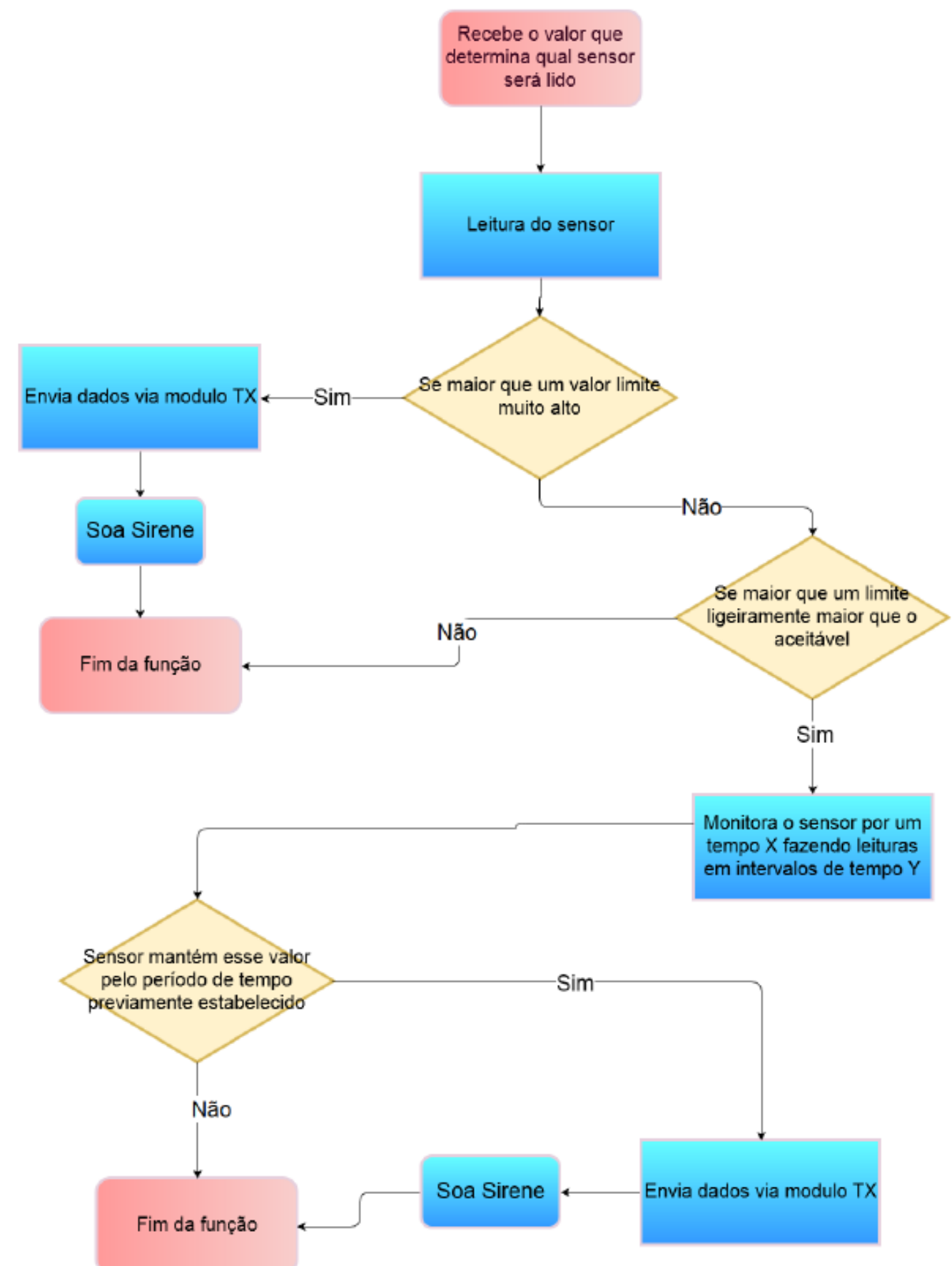

Fonte: (Autoria própria). 
Há também uma sub-rotina específica para avaliação da condição da carga da bateria, tanto do sistema embarcado na torre quanto da Casa de Telecom. Esta sub-rotina também consiste na leitura de um valor de sensor, no caso de tensão, e comparação com uma referência. $O$ valor medido é atualizado na IHM a cada uma hora em caso de operação normal ou instantaneamente quando o valor de tensão da bateria opera fora dos limites estipulados como seguro. O fluxograma da Figura 5 ilustra a sub-rotina descrita.

Figura 5 - Sub-rotina de avaliação da condição operativa dos bancos de bateria.

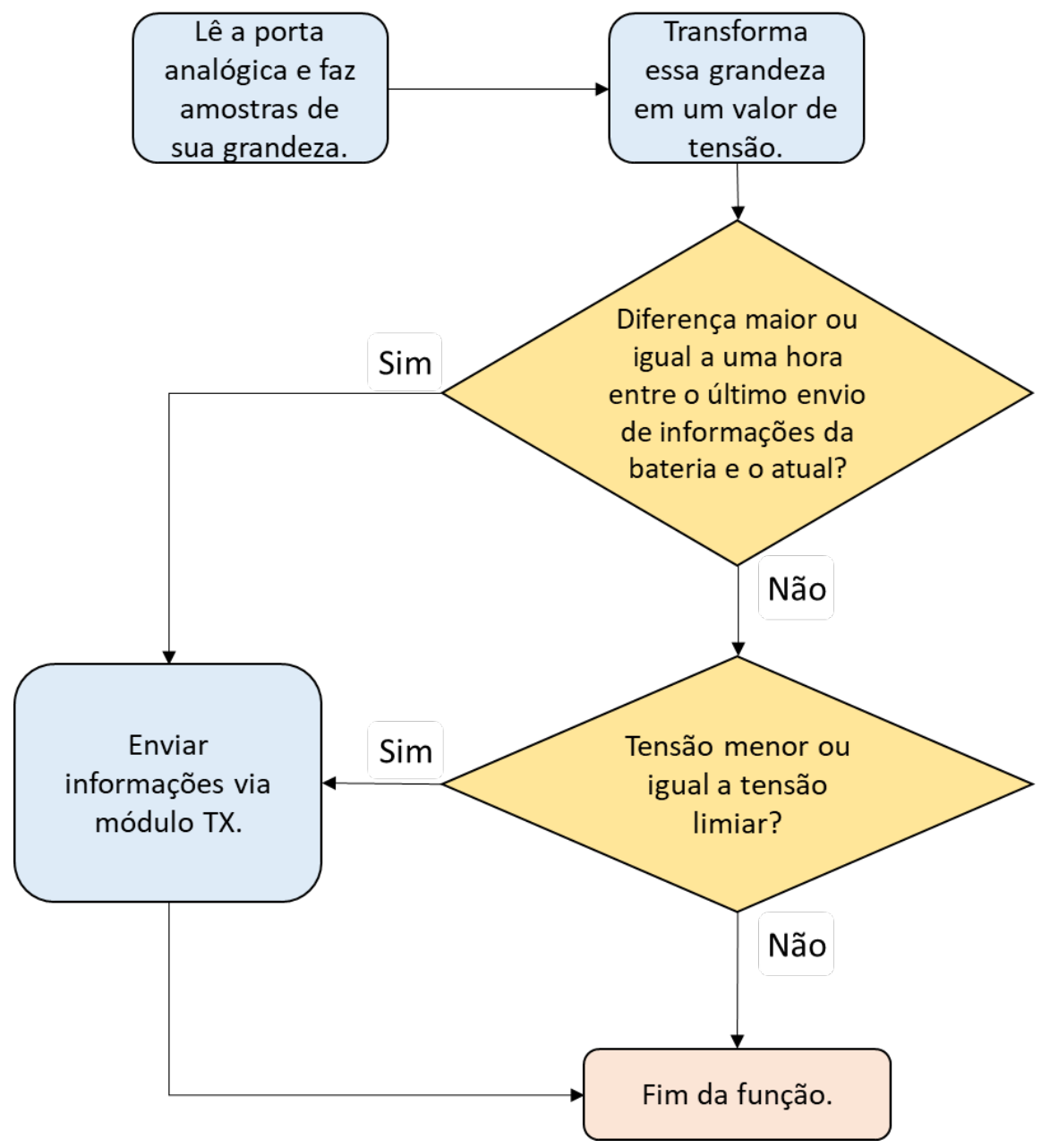

Fonte: (Autoria própria).

O microcontrolador localizado na Casa de Telecom é o responsável por receber as informações de dados dos estais, do banco de baterias embarcado na torre, monitorar o banco de baterias da Casa de Telecom e controlar as informações apresentadas na IHM. A Figura 6 ilustra as informações apresentadas pela IHM, localizada na Casa de Telecom. 
Figura 6 -Informações apresentadas na IHM.

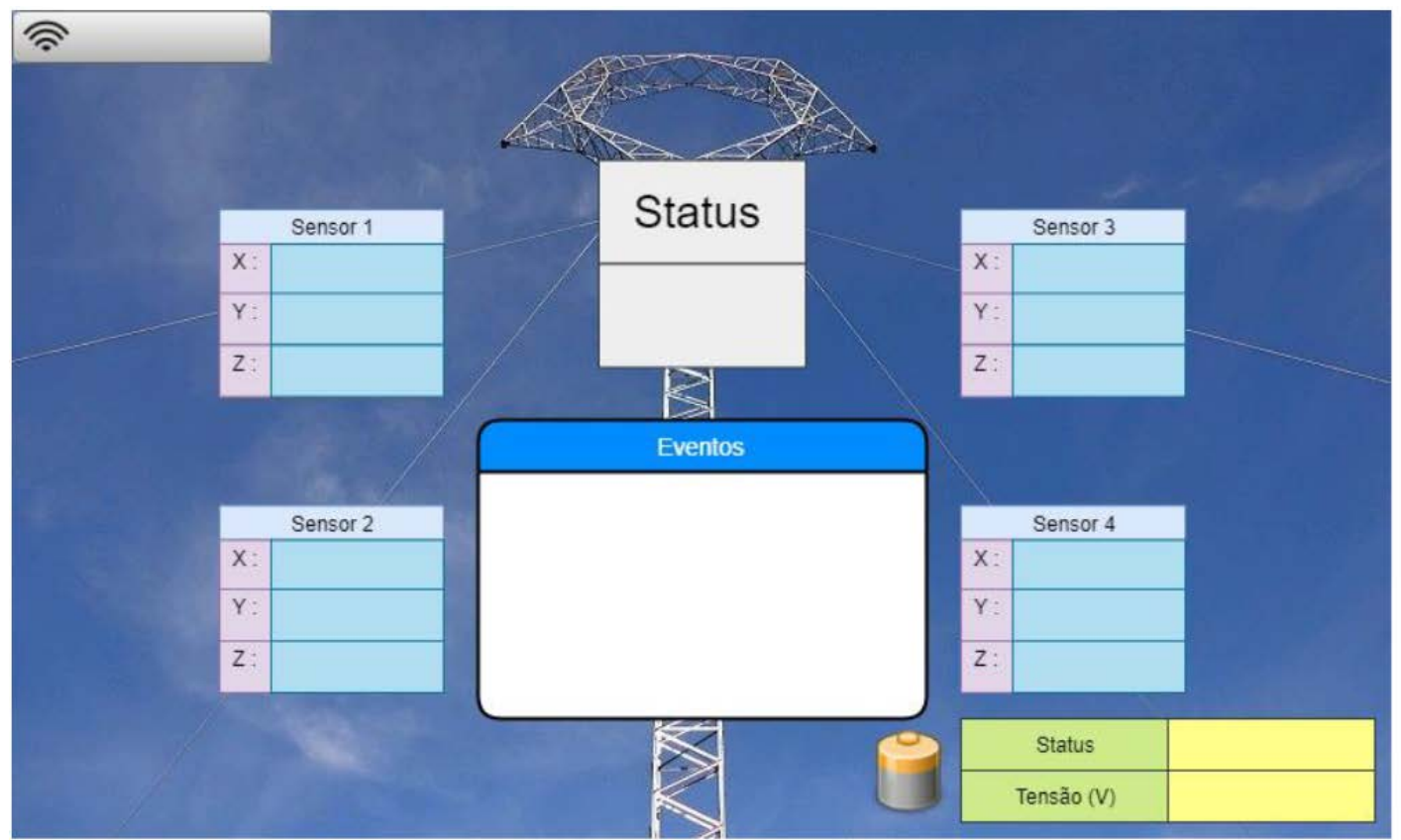

Fonte: (Autoria própria).

Nos campos "Sensor X" são apresentadas as medidas obtidas nos três eixos pelos acelerômetros instalados nos cabos estaiados. O campo "Status" indica se o modo de operação atual é normal ou se algum cabo está operando de forma anormal. No campo "Eventos" é possível visualizar um pequeno histórico com o último cabo em operação anormal e o tempo transcorrido desde o evento. No canto superior esquerdo é indicado se o sistema embarcado na torre e o localizado na Casa de Telecom estão conectados. Por fim, no canto inferior direito, são apresentados os dados referentes à bateria instalada na torre da linha de transmissão que, no caso, são a tensão medida em seus terminas bem como uma classificação de se a mesma se encontra carregada ou descarregada.

\subsection{TESTES LABORATORIAIS}

Uma bateria de testes foi realizada em ambiente controlado para levantamento da resposta dos sensores localizados nos estais das torres às diversas formas de operação à que os cabos são submetidos durante sua vida útil. Para esta etapa, foi construído um protótipo do sistema embarcado na torre, apenas com o mínimo necessário para realização de leituras do acelerômetro. Além disso, foram realizados testes para atestar a capacidade do sistema de comunicação de dados em termos de distância e qualidade do sinal enviado. Estes testes estão descritos nos itens $2.3 .1 \mathrm{e}$ 2.3.2. 


\subsubsection{Teste do Sensores}

Para possibilitar a correta classificação entre operação normal e anormal dos estais das torres de linha de transmissão faz-se necessário conhecer a resposta dos sensores às diferentes formas de operação possíveis à que estes cabos estão sujeitos. Os testes dos sensores e avaliação dos resultados foram extensivamente detalhados por (SIMÕES, 2018), onde também são apresentadas detalhadamente as condições consideradas na realização dos testes bem como a análise dos resultados obtidos.

Os ensaios realizados nesta etapa seguiram uma metodologia que consistia na repetição de 5 testes para cada uma das condições operativas, descartando medidas que apresentassem desvios de $25 \%$ em relação à média de todas as medidas obtidas. Nesta etapa, foram consideradas 5 formas de operação do cabo estaiado:

1. Operação normal;

2. Ventos de baixa intensidade e chuva fraca;

3. Circulação de veículos automotores;

4. Colisão de pássaros;

5. Colisões de grande energia;

6. Tentativa de corte do cabo.

Os resultados dos três primeiros testes foram semelhantes, caracterizados pela pequena variação no sinal gerado pelo acelerômetro. Os valores obtidos nos dos testes de colisão de pássaro, considerada de baixa energia, permitiu definir o limiar entre a operação normal e a operação anormal. O teste de colisão de grande energia foi preponderante para se estabelecer que o fundo de escala para o acelerômetro a ser utilizado deve ser da ordem de $8 g$. Por fim, no teste referente à tentativa de corte do cabo foi constatado que somente o valor da aceleração não é suficiente para classificar este tipo de operação anormal, sendo necessário também avaliar se há repetição do evento.

\subsubsection{Teste do Sistema de Comunicação}

Uma vez que o sistema embarcado detecta uma condição operativa anormal, é necessário que esta informação seja transmitida para o microcontrolador na Casa de Telecom que irá apresentar o resultado na IHM. Este sistema de transmissão de informação foi submetido à um teste 
em campo, no Parque Olímpico localizado na cidade do Rio de Janeiro - RJ. A Figura 7 ilustra o local em que o teste foi realizado e é possível observar destacado pela linha amarela a posição dos dois protótipos utilizados que, no caso, foi de $1 \mathrm{~km}$.

Figura 7 - Local do teste de comunicação com destaque para a posição dos protótipos.

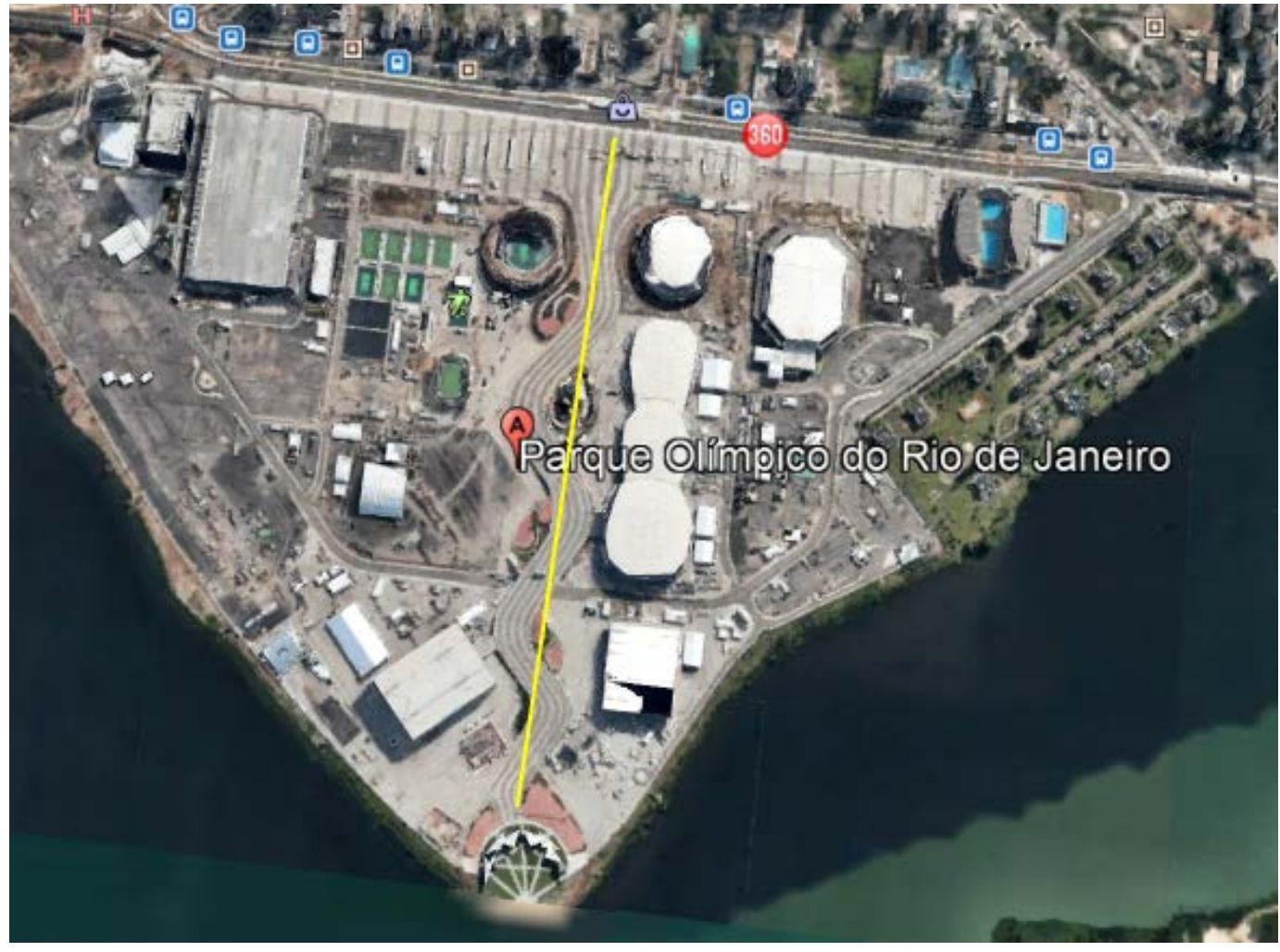

Fonte: Autoria própria.

A premissa utilizada foi a de realização de 5 balanços no sensor de forma a simular uma condição anormal de operação, suficiente para ser detectada pelo sistema embarcado, ao longo da operação de medições natural do sensor (conforme exposto na tabela 1).

Constatou-se que todos os balanços foram classificados corretamente como operação anormal pelo protótipo que representou aquele que ficará embarcado na torre e esta informação foi transmitida para o protótipo que simulou aquele localizado na Casa de Telecom. Todos os dados chegaram corretamente ao protótipo da Casa de Telecom, sem que houvesse perda de pacote de dados ou erro no envio ou na interpretação da informação recebida. Além disso, todos os eventos de operação anormal foram corretamente apresentados na IHM.

Desta forma, constatou-se que o sistema de comunicação entre os protótipos funciona corretamente na aplicação proposta.

Todos os equipamentos utilizados nos testes do sistema de telecomunicações foram alimentados pelo conjunto de painéis fotovoltaicos e banco de baterias, atestando também o 
correto funcionamento destes. Este conjunto é composto com duas baterias de $12 \mathrm{~V}$, com capacidade de 115 Ah, e duas placas solares fotovoltaicas de $270 \mathrm{~W}$ da fabricante Risen.

Tabela 1 - Dados Capturados em Ensaio.

\begin{tabular}{|c|c|c|c|c|c|}
\hline \multicolumn{3}{|c|}{$\begin{array}{l}\text { Leitura em cada eixo do } \\
\text { sensor }\end{array}$} & \multirow{2}{*}{$\begin{array}{c}\begin{array}{c}\text { Tempo de } \\
\text { Ensaio }\end{array} \\
\text { Tempo (ms) }\end{array}$} & \multirow{2}{*}{$\begin{array}{c}\text { Indicador de } \\
\text { Variação }\end{array}$} & \multirow{2}{*}{$\begin{array}{c}\text { Checagem de Operação } \\
\text { Anormalidade }\end{array}$} \\
\hline$x$ & $Y$ & $\mathbf{Z}$ & & & \\
\hline 488 & -6804 & 15022 & 72 & & FALSO \\
\hline 564 & -6656 & 15366 & 159 & 284 & FALSO \\
\hline 656 & -6808 & 1534 & 247 & 88 & FALSO \\
\hline 452 & -6956 & 15296 & 336 & 96 & FALSO \\
\hline 512 & -6724 & 15366 & 424 & 68 & FALSO \\
\hline 516 & -6852 & 15414 & 513 & 48 & FALSO \\
\hline 548 & -6644 & 15318 & 602 & 96 & FALSO \\
\hline 468 & -6748 & 15154 & 690 & 164 & FALSO \\
\hline 568 & -6912 & 15282 & 778 & 128 & FALSO \\
\hline 652 & -6612 & 15354 & 867 & 72 & FALSO \\
\hline 588 & -6632 & 15414 & 955 & 60 & FALSO \\
\hline 520 & -6848 & 15096 & 1043 & 316 & FALSO \\
\hline 560 & -6728 & 15346 & 1133 & 248 & FALSO \\
\hline 552 & -6860 & 15466 & 1222 & 120 & FALSO \\
\hline 568 & -6576 & 15560 & 1311 & 216 & FALSO \\
\hline 5 & -6736 & 15244 & 1401 & 24 & FALSO \\
\hline 432 & -6852 & 15218 & 19667 & 36 & FALSO \\
\hline 680 & -6752 & 15330 & 19758 & 112 & FALSO \\
\hline 528 & -6736 & 15442 & 19849 & 112 & FALSO \\
\hline 620 & -6760 & 15098 & 19939 & 344 & FALSO \\
\hline 3244 & -32368 & -31862 & 20033 & 46960 & VERDADEIRO \\
\hline 944 & -32369 & -31283 & 20126 & 579 & FALSO \\
\hline 3200 & -12460 & 31674 & 20218 & 62957 & VERDADEIRO \\
\hline 7064 & 16856 & 29382 & 20311 & 2292 & FALSO \\
\hline-3204 & -20712 & -31282 & 20405 & 60664 & VERDADEIRO \\
\hline-1024 & 5104 & -16054 & 20591 & 29840 & FALSO \\
\hline-3132 & -20292 & 23654 & 20684 & 39708 & VERDADEIRO \\
\hline-1892 & -8160 & 24162 & 20777 & 508 & FALSO \\
\hline-3100 & -16020 & 778 & 20869 & 23384 & FALSO \\
\hline 96 & -18652 & -31482 & 20960 & 32260 & VERDADEIRO \\
\hline 208 & 1768 & -2458 & 21142 & 30932 & FALSO \\
\hline-1820 & -8824 & 3218 & 21234 & 5676 & FALSO \\
\hline 74 & -8784 & -422 & 21323 & 3640 & FALSO \\
\hline 72 & 4484 & 2378 & 21411 & 2800 & FALSO \\
\hline-304 & -18192 & 23098 & 21505 & 20720 & FALSO \\
\hline 560 & -11768 & 26874 & 21596 & 3776 & FALSO \\
\hline
\end{tabular}


Na tabela 1, os resultados obtidos ao longo de um ensaio são exibidos, sendo os momentos de perturbação intencional destacado em amarelo. Nestes dados, a medição dos eixos é obtida em valor numérico referente ao máximo de 16 bits em proporção linear à escala de tensão medida, sendo 32.768 equivalente a $5 \mathrm{~V}$ e -32.768 equivalente a $0 \mathrm{~V}$, conforme mencionado na seção 2.1.

\subsection{INSTALAÇÃO EM CAMPO E COMISSIONAMENTO}

O resultado positivo dos testes laboratoriais levou para a próxima etapa, que consistiu em instalar o protótipo desenvolvido em uma das instalações de transmissão da Norte Brasil Transmissora de Energia bem como seu comissionamento.

A torre escolhida para instalação do sistema embarcado foi a $n^{\circ} .1479 / 2$ do Bipolo 600 kV $D C$ Porto Velho / Araraquara. A Cada de Telecom localiza-se a uma distância de 45 metros da torre.

\subsubsection{Sistema Embarcado na Torre}

A torre 1479/2, conforme preconizado no projeto e ilustrado nas Figura 7 e 8 , do tipo estaiada com quatro estais.

Os serviços realizados para o sistema embarcado na torre consistiram em fixar um sensor em cada cabo estaiado, totalizando quatro sensores, e instalar na estrutura da torre o banco de baterias, os painéis fotovoltaicos e o quadro elétrico em que ficam os componentes associados a esta parte do sistema. Na Figura 10 estão destacados em azul o quadro elétrico onde ficam ao

Figura 8 - Torre 1479/2 com destaque para os quatro estais.

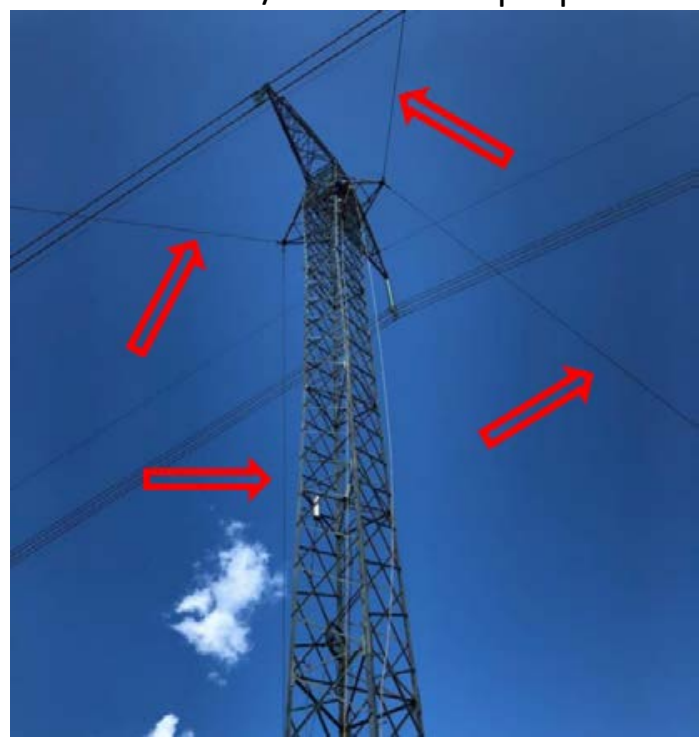

Fonte: Autoria própria. 
Figura 9- Torre 1479/2 com destaque para os quatro estais.

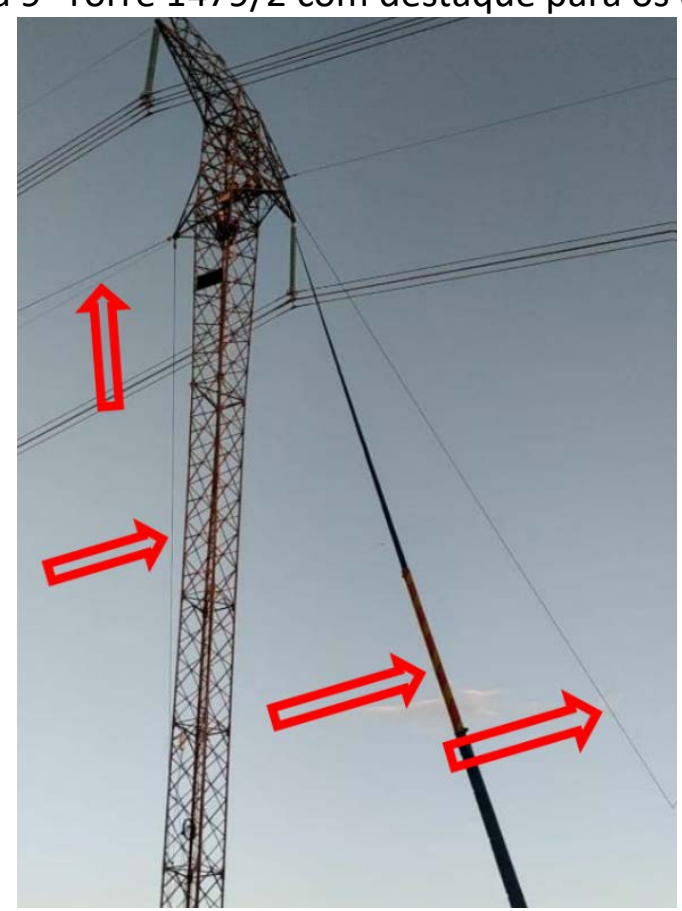

Fonte: Autoria própria.

Arduino e demais equipamentos de controle, em verde está destacado o quadro elétrico das baterias e em amarelo as placas solares fotovoltaicas.

Figura 10 - Sistema embarcado na torre. Destaque em vermelho para o microprocessador.
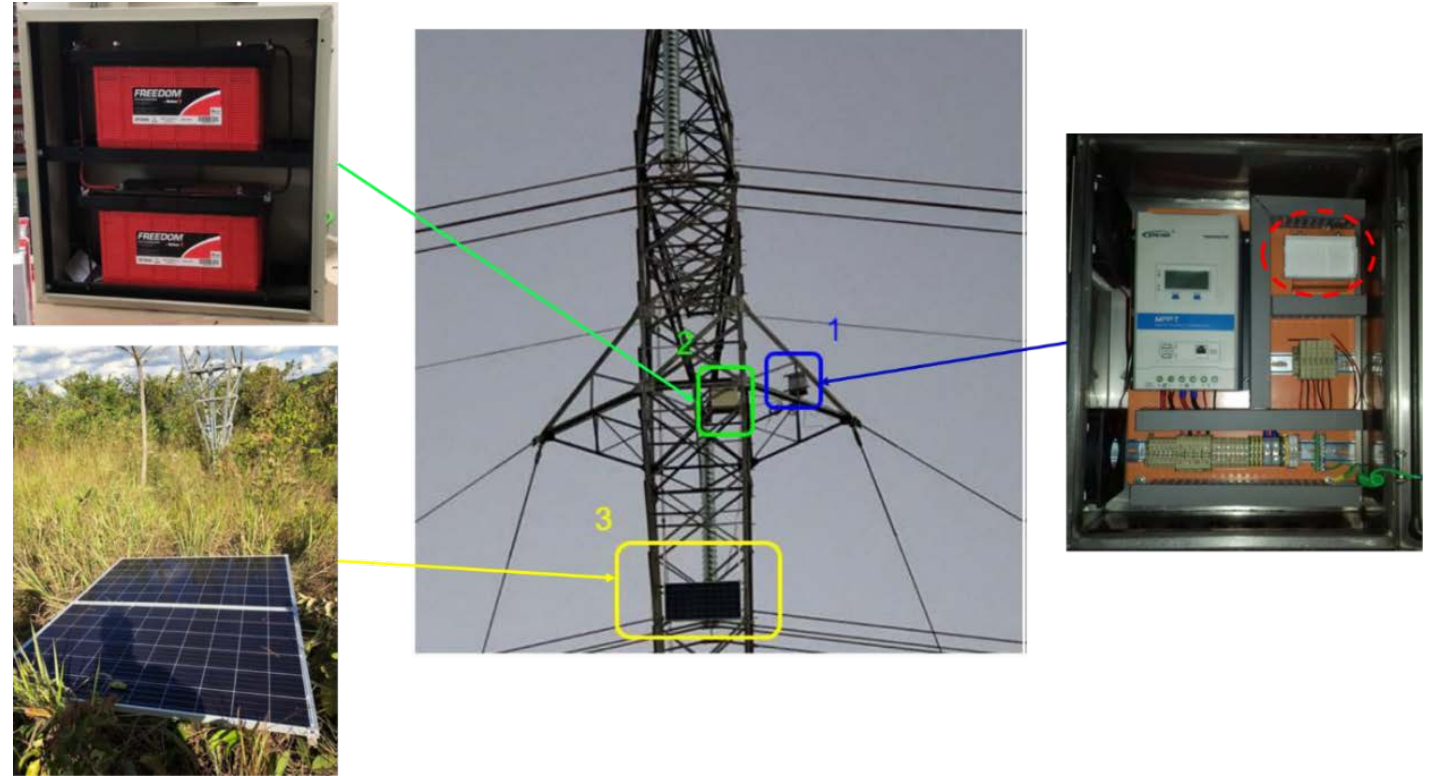

Fonte: Autoria própria. 


\subsubsection{Sistema Instalado na Casa de Telecom}

O quadro elétrico com a IHM, ilustrado na Figura 11, foi instalado na Casa de Telecom mais próxima da torre 1479/2. Nesta figura está apresenta uma visão do interior do painel e a visão frontal do painel, em que se observa a IHM.

Figura 11 - Sistema instalado na Casa de Telecom.
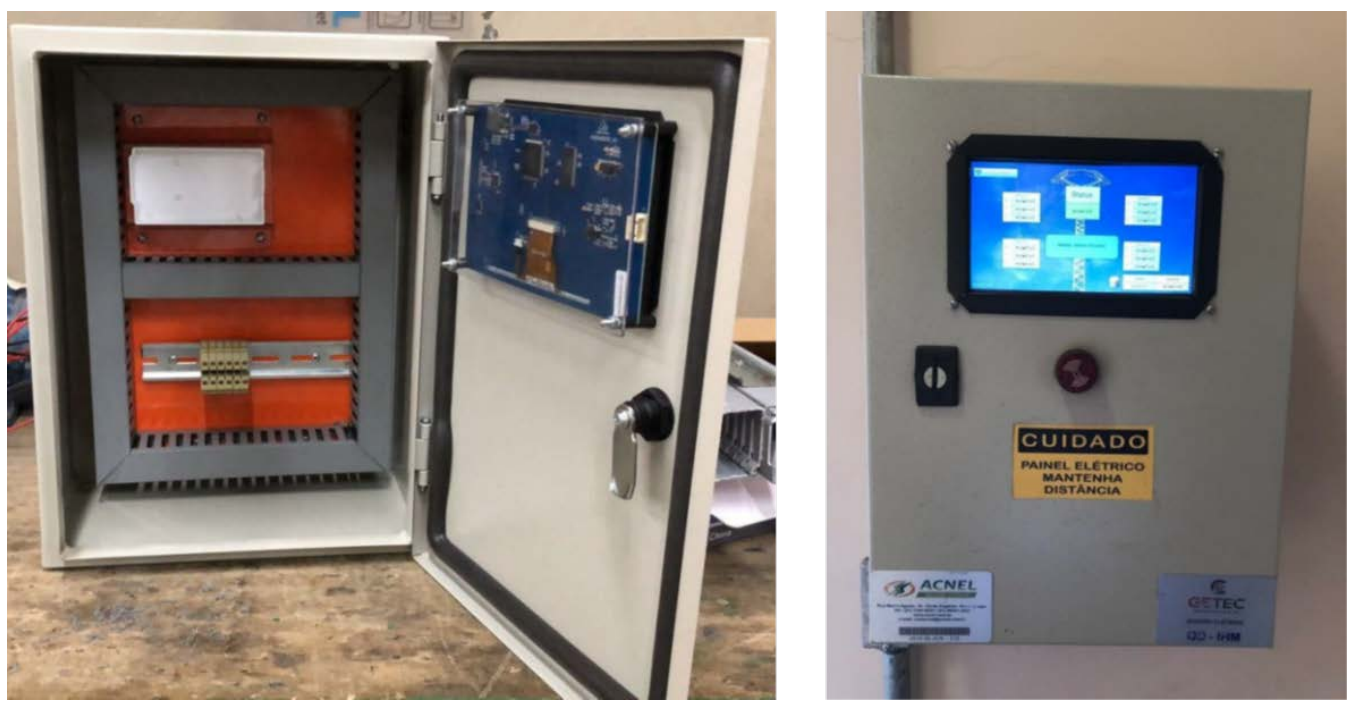

Fonte: Autoria própria.

O microprocessador localizado neste quadro elétrico é responsável por receber as informações do sistema embarcado na torre da linha de transmissão e apresentá-las na IHM.

\subsubsection{Testes Locais e Comissionamento}

Uma rotina de testes semelhante aos descritos no item 2.2 foi realizada no sistema após a sua instalação para atestar o correto funcionamento do protótipo. Os testes consistiram em verificar se as informações apresentadas na IHM eram condizentes com o modo de operação em que os cabos se encontravam, garantindo assim que o sistema embarcado era capaz de identificar a situação de risco, transmitir para o sistema na Casa de Telecom e apresenta-la na IHM.

O primeiro teste consistiu em verificar se o sistema era capaz de identificar corretamente o modo de operação normal, ou seja, aquele em que as vibrações presentes nos cabos correspondem à sua frequência natural livre de perturbações externas. Este teste não produziu variações significativas nos valores dos sensores e, portanto, não foi classificado pelo sistema como operação anormal. A informação apresentada na IHM é a apresentada na Figura 12. 
Figura 12 - Informação apresentada na IHM para um caso de operação normal.

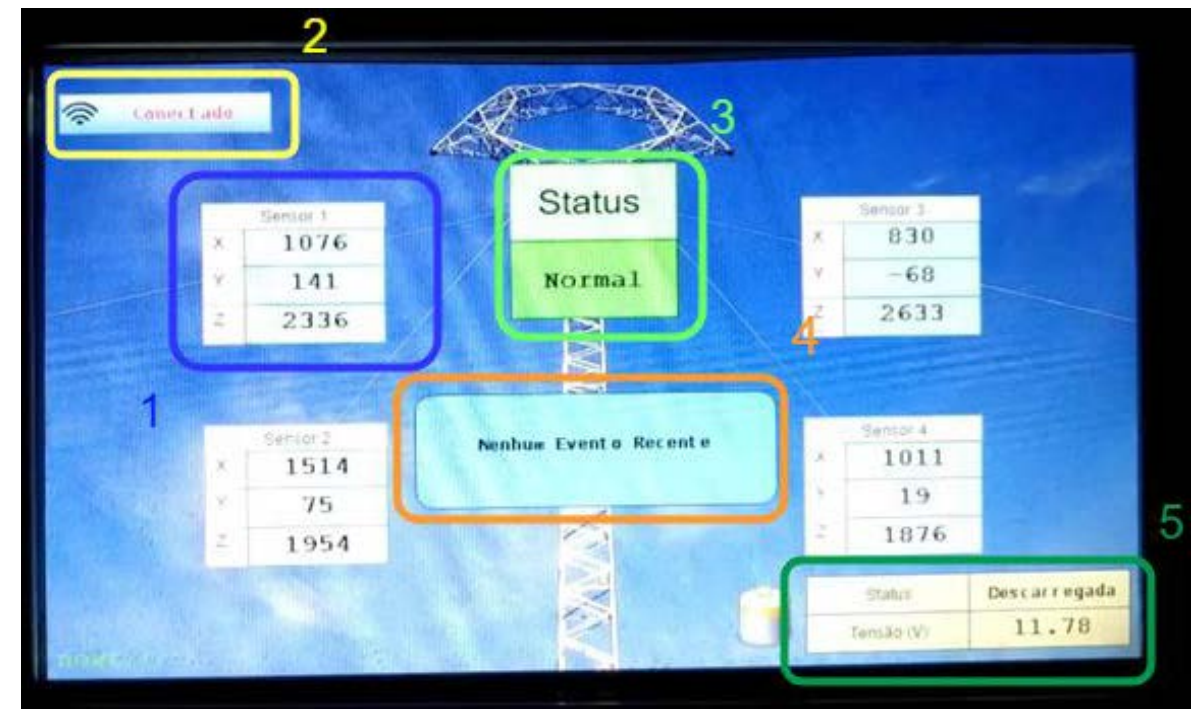

Fonte: Autoria própria.

No segundo teste, um dos estais foram submetidos à repetidas vibrações mecânicas de menor energia, apresentando um modo de operação com assinatura de frequência compatível com aquela obtida na tentativa de serrar o cabo. Estas vibrações foram produzidas manualmente empurrando e puxando o cabo em um ponto próximo do seu ancoramento no chão e foram corretamente sinalizadas na IHM. A Figura 13 ilustra o teste realizado e sua sinalização na IHM com destaque para a iluminação indicando situação de risco ao cabo.

Figura 13 - Aplicação e resultado do segundo teste em campo.
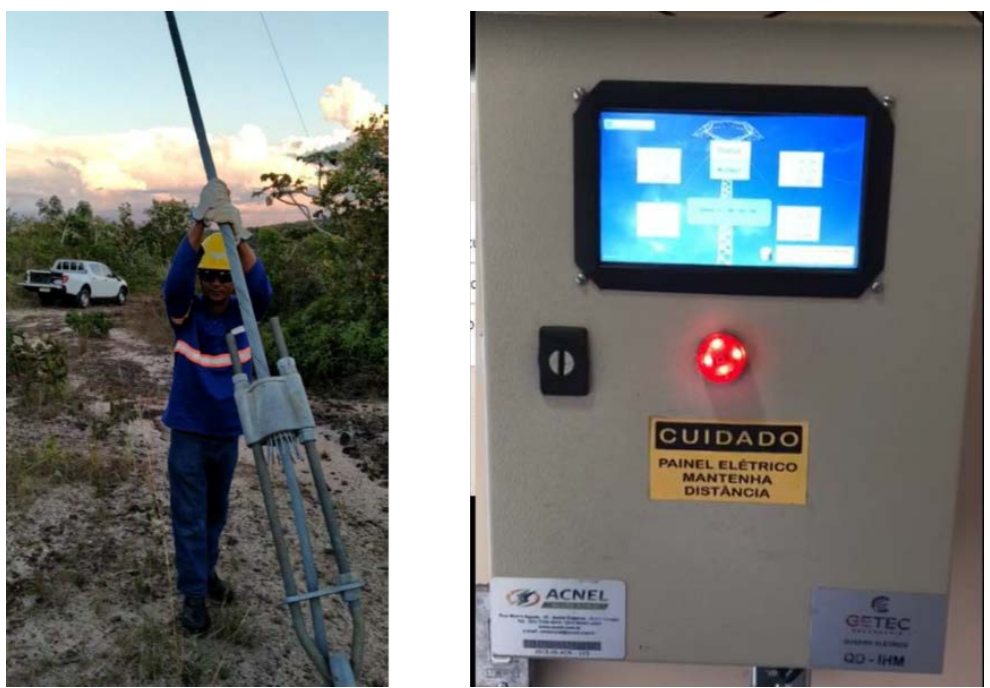

Fonte: Autoria própria. 
O terceiro e último teste realizado consistiu na aplicação de vibrações mecânicas de grande energia por meio da aplicação de golpes de marreta no cabo. Este tipo de operação anormal também foi corretamente classificado e apresentado na IHM localizada na Casa de Telecom. Observa-se na Figura 14 as informações apresentadas na IHM, onde está registrado a perturbação detectada pelo sensor localizado no cabo identificado como $n^{\circ} 2$ ocorrida há 9 segundos.

Figura 14 - Informação apresentada na IHM logo após uma operação anormal.

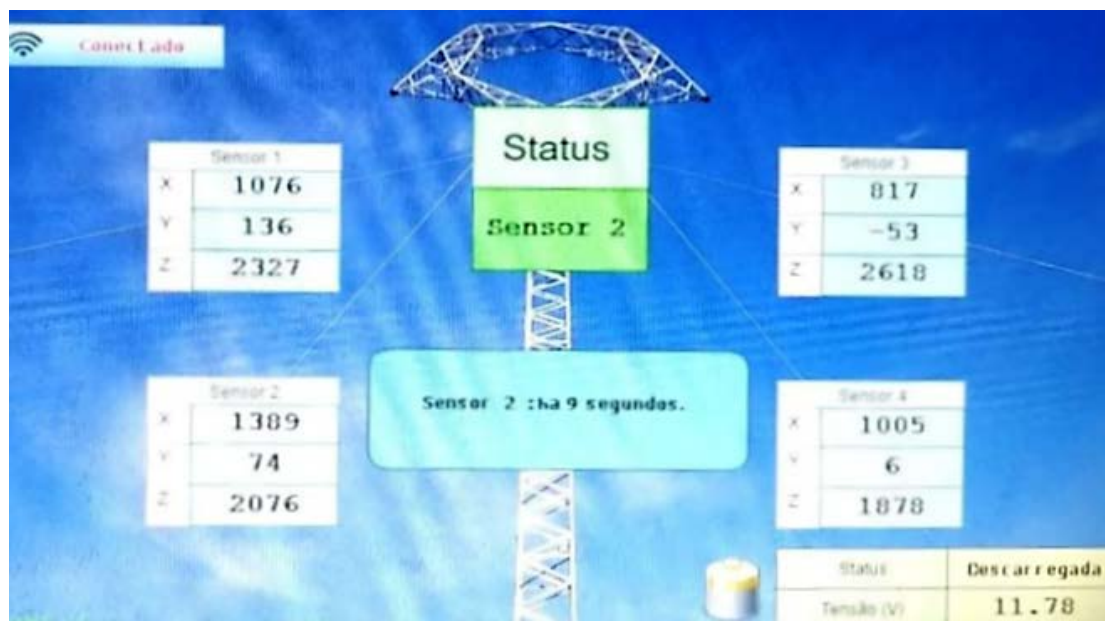

Fonte: Autoria própria.

Em todos os restes realizados, houve a correta classificação do modo de operação dos cabos estaiados e envio satisfatório dos dados pelo sistema de comunicação.

\section{CONSIDERAÇÕES FINAIS}

Foram apresentados os principais componentes de um sistema de um sistema inteligente de detecção e monitoramento para evitar sinistros com queda de torres de transmissão de energia. Também foram apresentados e discutidos os resultados dos testes laboratoriais e comissionamento dos equipamentos em campo.

Tanto os testes laboratoriais quanto em campo realizados nos sensores foram satisfatórios do ponto de vista da classificação correta do modo de operação dos cabos estaiados, se normal ou anormal, o que comprova que os tipos e modelos de sensores especificados são apropriados para a aplicação em curso.

No que se refere ao sistema de comunicação entre sistema embarcado na torre e o localizado na Casa de Telecom, foi constatado após exaustivos testes que o sistema proposto é capaz 
de realizar a troca de dados por até $1 \mathrm{~km}$ em campo aberto sem que ocorra perda na qualidade da comunicação.

As informações apresentadas ao usuário na IHM são simples e intuitivas. Nos testes em campo foi possível constatar a correta apresentação das informações, permitindo inferir que todas as funcionalidades do sistema desenvolvido funcionam corretamente para a aplicação em questão.

Em projetos futuros, propõe-se a utilização da rede de comunicação da concessionária de transmissão para enviar as informações da Casa de Telecomunicações para o Centro de Operação do Sistema - COS ou alguma outra localidade e dispositivo associados à empresa de transmissão. Será possível, desta forma, realizar uma avaliação sistêmica da natureza dos eventos identificando, por exemplo, se várias torres foram derrubadas, o que pode ter origem em fortes vendavais, ou se somente uma ou poucas torres foram derrubadas, que pode ter origem em ações de vandalismo.

O estudo dos sistemas solar e eólico mostrou que a SE Morro do Chapéu II possui boas condições para aproveitar a complementariedade da geração de energia proveniente das duas fontes, sendo este local ideal para aplicação de um protótipo para avaliação da performance do sistema de controle. Foi evidenciado que há uma gama de equipamentos disponíveis no mercado capazes de atender os critérios estabelecidos.

\section{AGRADECIMENTOS}

À Norte Brasil Transmissora De Energia S.A. - NBTE, que propôs e financiou, na forma de um Projeto de P\&D da ANEEL, este trabalho.

\section{REFERÊNCIAS}

CLARO, R. T., et al. Sistema de segurança patrimonial aplicado a Linhas de transmissão de energia elétrica. XV SNPTEE, Recife - PE, 2009.

GETEC ENGENHARIA. Desenvolvimento de um sistema inteligente de detecção e monitoramento para evitar sinistros com queda de torres de transmissão de energia. Rio de Janeiro, 2017.

HUBACK, V. B. S. Medidas ao combate a perdas elétricas não técnicas em áreas com severas restrições à operação de sistemas de distribuição de energia elétrica. Dissertação de Mestrado, UFRJ. Rio de Janeiro, 2018.

LIMA, R. A. Análise e Gestão de Riscos das Ocupações de Faixas de Linhas de Transmissão: Estudo de Caso da Vila Alta Tensão. Universidade Federal de Ouro Preto, Ouro Preto- MG, 2012. Operador Nacional do Sistema Elétrico - ONS. 2019. Disponível em: <http://ons. org.br/>. [Online, acessado em 03/02/2019]. 
RAUPP, Fabiano Maury; DA SILVA, Ana Cristina. Custos em Geração de Energia: Evidências empíricas a partir do Complexo Termelétrico Jorge Lacerda. Revista Sodebras [on line]. v. 11, n.126, junho/2016, p. 17-22. ISSN 1809- 3957. Disponível em:

http://www.sodebras.com.br/edicoes/N126.pdf. Acesso em 01/07/2019.

ROSSI, J. A. D., et al. Sistema de Transmissão de Dados para Monitoramento de Linhas de Transmissão da Eletronorte. V CITENEL - Congresso de Inovação Tecnológica em Energia Elétrica, Belém - PA, 2009.

SIMÕES, Tallis Amorim, SILVA, Alex de Lima, Neves, Fabrício, Silva, José Alves Junior.

Desenvolvimento de um sistema inteligente de detecção e monitoramento para evitar sinistros com queda de torres de transmissão de energia. Revista Sodebras [on line]. v. 13, n.153, setembro/2018, p. 108-112. ISSN 1809- 3957. Disponível em:

http://www.sodebras.com.br/edicoes/N153.pdf Acesso em 25/05/2018.

TKD INVESENSE. MPU-6000/MPU-6050 Product Specification. Disponível em:

https://www.invensense.com/products/motion-tracking/6- axis/mpu-6050/. Acesso em 01/07/019. 